\title{
Experimental investigation on HSFP using MWCNT based nanofluids for high power light emitting diodes
}

\author{
Sangmesh B ${ }^{1,2 *}$, Gopalakrishna K. ${ }^{1}$, Manjunath S.H. ${ }^{2}$, Kathyayini N. ${ }^{1}$, K. Kadirgama ${ }^{3 *}$, \\ M. Samykano ${ }^{3 *}$, G.C. Vijayakumar ${ }^{1}$ \\ ${ }^{1}$ Centre for Incubation, Innovation, Research and Consultancy, Jyothy Institute of Technology, \\ Bangalore, Karnataka, India \\ ${ }^{2}$ Research Scholar, VTU, Belagavi, Karnataka, India \\ *Email: sangmesh.sangu293@gmail.com \\ Phone: +91 9964311824; Fax: +91 448797660 \\ ${ }^{2}$ School of Mechanical Engineering, Reva University, Bangalore, Karnataka, India \\ ${ }^{3}$ Faculty of Mechanical Engineering, Universiti Malaysia Pahang, 26600 Pekan, Pahang, Malaysia \\ *Email: kumaran@ump.edu.my; mahendran@ump.edu.my
}

\begin{abstract}
LEDs, of late, have received attention as the next generation lighting system for enhanced luminous efficiency and higher lifespan. However, the thermal management of the LEDs is the crucial parameter to be countered for global acceptance as a revolutionary illumination source. This paper reports the experimental investigation of natural convective heat transfer of high power LED COBs using MWCNT and MWCNT-CuO nanofluids mixed with de-ionized water. The study uses MWCNT based nanofluids as a route to enhance the heat transfer of high power LEDs by the passive cooling technique. This study presents an innovative cooling device integrated with numerous fluid pockets, called the HSFP, to achieve the enhanced thermal performance of heat sinks for applications in high intensity LED lights. Nanofluids of various concentrations were formulated and their heat transfer performance evaluated using a series of experiments and compared with liquid cooling and a conventional heat sink. The experimental finding reveals 20 $30 \%$ lowered thermal resistance using the new HSFP (nanofluids). Thus, the HSFP found to effectively dissipates the heat in high-power LED COBs using nanofluids as the cooling medium compared to the conventional heat sink.
\end{abstract}

Keywords: HSFP; Heat Sink with fluid pockets; Thermal management of LEDs; Nanofluids.

\section{INTRODUCTION}

Being ten (10) times more efficient than incandescent lights, LEDs are increasingly penetrating in domestic applications. Their increased reliability, coupled with long operating life, renders them as an obvious choice for next-generation lighting systems. However, most of the power supplied to them converts to heat. The increased input power further cause's degradation of luminance efficiency and lifespan since higher heat is generated. Therefore, it is essential to have a proper heat dissipation module to ensure proper operation. Hence, thermal management of LEDs is the crucial parameter to enhance their lifespan and luminance effect. Active or preferably passive cooling with air can be the predominant choice for LED lighting systems. A proposed design must have a meager thermal resistance between the junction and the heat sink base which relies on conduction heat transfer [1]. Tisha Dixit, et al. [2] studied the miniaturization of the heat sink and found that characteristics such as compactness, small size and lesser weight attracted widespread attention. 
Yiwei Wang, et al. [3] studied a new flat plate heat pipe with a micro-fins cast on the condensation surface. It was found to be more effective for heat dissipation of high-power LED COBs than the traditional new flat plate heat pipe. Z. Lin et al. [4] developed a plate oscillating heat pipe heat sink for LED cooling. They found that when a plate OHP was used in an LED heat sink, it led to a significant decrease in the temperature of the LED through a natural convection process. Whereas, the thermophysical properties and heat transfer performance have been carried out by Patrice Estell et al. [5]. Hamed Khajeh Arzani, et al. [6, 7] studied MWCNT-ASP (aspartic acid) with water for different weight fractions experimentally.

Their results showed a significant increase in heat transfer rate. M. Hemmat Esfe et al. [8] studied the thermo-physical properties of nanofluids and concluded that thermal conductivity increases with an increase in temperature. Meanwhile, W.S. Sarsam et al. [9] revealed that aqueous colloidal dispersions of $\beta$-alanine-MWCNTs are promising alternative working fluids for enhanced thermal performance. B. Munkhbayar et al. [10] performed an investigation on the enhancement of thermal conductivity of silver nanoparticle with multi-walled carbon nanotubes. They reported that grinding has significantly improved the dispersion stability and thermal conductivity of MWCNT. S Jyothirmayee Aravind et al. [11] reported that no surfactant, good fluidity, long-term stability, and high thermal conductivity would enable f-MWCNT nanofluids to be used as advanced coolants in thermal management applications. Sh. M. Vanaki et al. [12] observed that nanofluids have the ability to significantly enhance heat transfer rate of conventional heat transfer fluids as a result of the dispersed highly conductive nanoparticles in the base liquid. Mohammadali Baghbanzadeh et al. [13] studied the effect of hybrid silica nanosphere/MWCNT on the thermal conductivity of distilled water.

Their results showed that the effective thermal conductivity of nanofluid was obtained by increasing the concentration of nanoparticles. K. Kouloulias et al. [14] performed an experimental study on natural convection of $\mathrm{Al}_{2} \mathrm{O}_{3}-\mathrm{H}_{2} \mathrm{O}$. They found that the convective heat transfer is enhanced when the applied heat flux increases and the induced flow becomes more turbulent. Zeinali Heriset al. [15] studied $\mathrm{Al}_{2} \mathrm{O}_{3}$ /water nanofluid heat transfer in laminar flow. Experimental investigations depict that heat transfer coefficient increases by increasing the volume fraction of nanoparticles in the nanofluid. Literature shows enhancement in the thermal conductivity, viscosity, and heat transfer through the use of nanofluids which increases with increasing volume of nanoparticles [16,17]. Y. Ding et al. [18] observed that enhanced convective heat transfer was obtained in comparison with pure water. It was also reported that the enhancement depends on the flow condition, concentration and the $\mathrm{pH}$ level. In another study of $\mathrm{Cu}-\mathrm{Ti}$ [19] with $\mathrm{Vol} \%$ of MWCNTs, it was reported that linear and small coefficient of thermal expansion was obtained for low concentrations of multiwall carbon nanotubes. In an experimental investigation by Majid Zarringhalam et al. [20] who studied the effect of volume fraction on heat transfer coefficient. They reported that heat transfer coefficient of nanofluids was higher than that of base fluid. It was also observed that the heat transfer coefficient and Nusselt number of nanofluids increases with increasing nanoparticles volume fraction.

The experimental study by Saeednia et al. [21] on $\mathrm{CuO}$ nanofluid for different concentrations, flowing inside a circular tube, showed that heat transfer coefficient increases with increasing nanoparticle concentration. Ali Hassan et al. [22] observed that enhancement of convective heat transfer coefficient of ethylene glycol (EG) base cuprous oxide $\left(\mathrm{Cu}_{2} \mathrm{O}\right)$ nanofluids was higher than for the base fluid. Hooman Yarmand et al. [23] prepared a hybrid nanofluid mixture of graphene nanoplatelet (GNP) and Platinum (Pt) nanofluids. Experimental results revealed that nanofluids have a higher heat transfer capability compared with the base liquid, and the improved heat transfer coefficient is dependent on Reynold number, and the weight concentration of the nanocomposite. Hong Seok Jo et al. [24] fabricated an innovative fractal-like $\mathrm{Cu}_{2} \mathrm{O}$ surface on a $\mathrm{NiCr}$ wire to identify the effects of wettability on pool boiling performance. Their results revealed that hydrophobic texturing is desirable for electronics cooling. R. Ellahi et al. [25] studied the nanolayer effects on SWCNTs and MWCNTs with natural convection. They observed that the 
velocity decreased by increasing the volume fraction of nanotubes. S. Zeinali Heriset al. [26] has studied $\mathrm{Al}_{2} \mathrm{O}_{3} /$ water nanofluid heat transfer in laminar flow. Their investigations depicted that heat transfer coefficient increased with an increase in the volume fraction of nanoparticles in nanofluid. I.D. Garbadeen et al. [27] performed experimental investigations on nanofluids consisting of multiwalled carbon nanotubes with water as the base fluid. Their results indicated that natural convection yielded a maximum enhancement in heat transfer performance of $45 \%$ at a volume concentration of $0.1 \%$.

This research supports the idea that nanofluids with effective thermal conductivity are higher than the thermal conductivity of the base fluid. Hina Muryam et al. [28] compared gold and silver nanoparticles with de-ionized water by studying their characteristics of convective heat transfer. They concluded that gold/DIW based nanofluids exhibit better convective heat transfer than silver/DIW based nanofluids. Till date, a number of experiments work has been carried out on forced convection. As noted from the literature, most of these studies involve flow rate, volume fraction and thermal conductivity rather than the heat transfer characteristics of nanofluids. A large number of studies have also been undertaken in improving the thermal conductivity of the nanofluids. Therefore, an attempt is made in the present study to fill this space with a focus on natural convection. Heat dissipation capabilities of nanofluids have been carried out and verified by natural convective heat transfer experiments for both steady state and transient conditions. An experimental setup was designed and fabricated for this purpose and applied to cool high-power LEDs. The junction temperature of the LEDs was measured to investigate enhanced heat transfer. The thermal resistance was analyzed to understand the cooling potential of nanofluids and liquid cooling better.

\section{METHODS AND MATERIALS}

\section{Materials}

In this work, a heat sink with fluid pockets (HSFP) was developed with parallel rectangular fins and multiple holes at the base. The novel HSFP was designed, fabricated and applied to cool highpower LED COBs. The holes were filled with nanofluids for effective heat dissipation from the LED junction. The main objective of the study was to investigate the influence of nanoparticles suspended in the base fluid on the heat transfer characteristic of nanofluids. MWCNT and MWCNT-Copper oxide (CuO-MWCNT) nanofluids with de-ionized water of various concentrations were used in the test. Due to their unique thermal properties, carbon nanotubes (MWCNTs) and MWCNT-CuO have been used as additives to increase thermal conductivity and other thermal properties of the nanofluids. The fluid pockets were filled with nanofluids along with the base fluid in wt $\%$ basis for different volume fraction. Measurement of heat transfer performance of MWCNT/DI water and MWCNT-CuO/DI watered nanofluids with nanoparticle concentration in the range of 0.2 to $0.8 \mathrm{wt} \%$ was tested and compared with liquid cooling and the conventional heat sink. Tests were done using stable nanofluids under natural convection condition for both steady state and transient conditions. The composite nanofluid has an equal volume of carbon nanotubes and copper in the base fluid. The nanofluids circulate within the fluid pockets by thermal energy and gravity force. Typical dimensions of the heat sink are shown in Figure 1. The aluminum heat sink was designed to suit actual street light applications. The heat sink was designed for a length of $200 \mathrm{~mm}$, the width of $120 \mathrm{~mm}$, the base of $10 \mathrm{~mm}$ height, and a fin height of $10 \mathrm{~mm}$ with a hole of diameter $6 \mathrm{~mm}$ drilled across the length to fill liquid with nanoparticles into it. The fins were maintained for half the length in order to achieve condensation of heat transfer fluids $[29,30]$. 


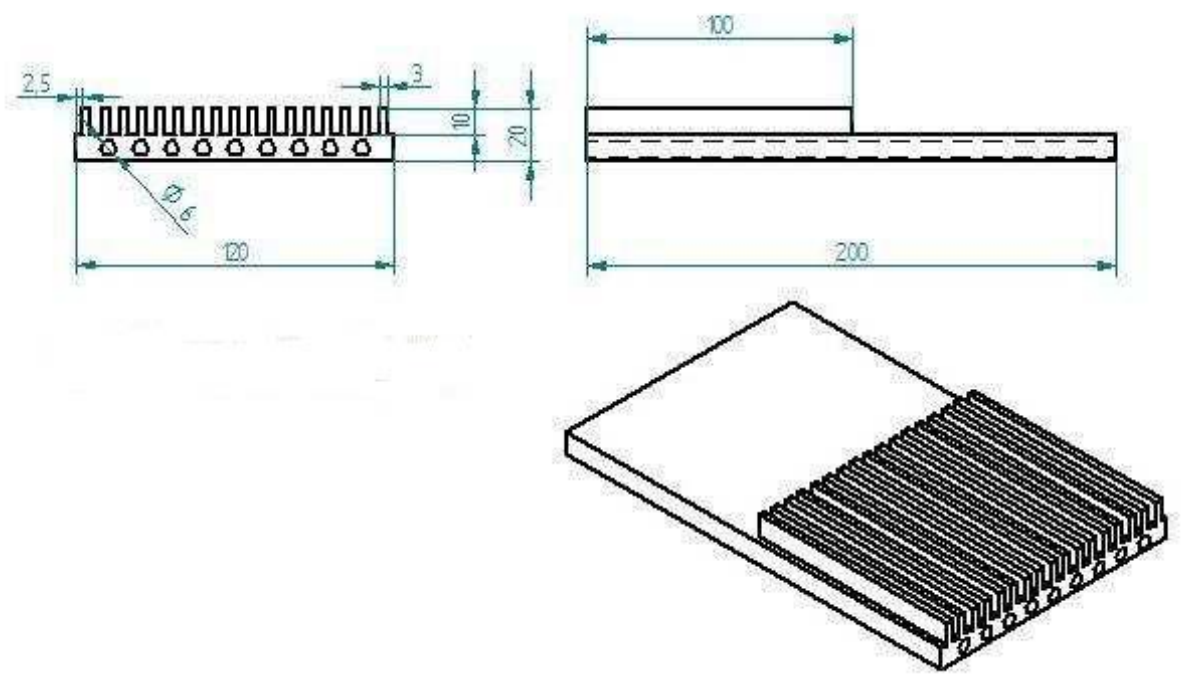

Figure 1. Heat Sink with fluid pockets and half-length fins

The LED setup chosen in this study has 3sets of COB LEDs of each having 40W (CREE Make- X lamp XP-G) with the wattage of $120 \mathrm{~W}$. A thermal interface material was used to establish proper surface contact to avoid air gap. The data acquisition system (TRACER make) has a resolution of $10^{-5}{ }^{\circ} \mathrm{C}$ to measure the junction temperature of the LED. The sampling rate was $1 / \mathrm{s}$. The thermocouple positions were diagnosed with the use of an infrared thermal image camera. Recognized hotspots over the MCPCB were placed with $\mathrm{T}$ type thermocouples to measure the junction temperature. Five trials were conducted for each test condition for repeatability of the measurements and to avoid uncertainties in the course of experimentation. Validation of the setup was done with the conventional heat sink of same configurations excluding fluid pockets. The test duration was $100 \mathrm{~min}$. Steady state was attained around 60 minutes of the experiment. The constant power input was $120 \mathrm{~W}$. Two different nanofluids such as MWCNT and CuO-MWCNT were used with different solid volume fractions of suspended nanoparticles. The test results were compared with liquid cooling and conventional heat sink. Figure 2 shows the experimental setup used in this study. 


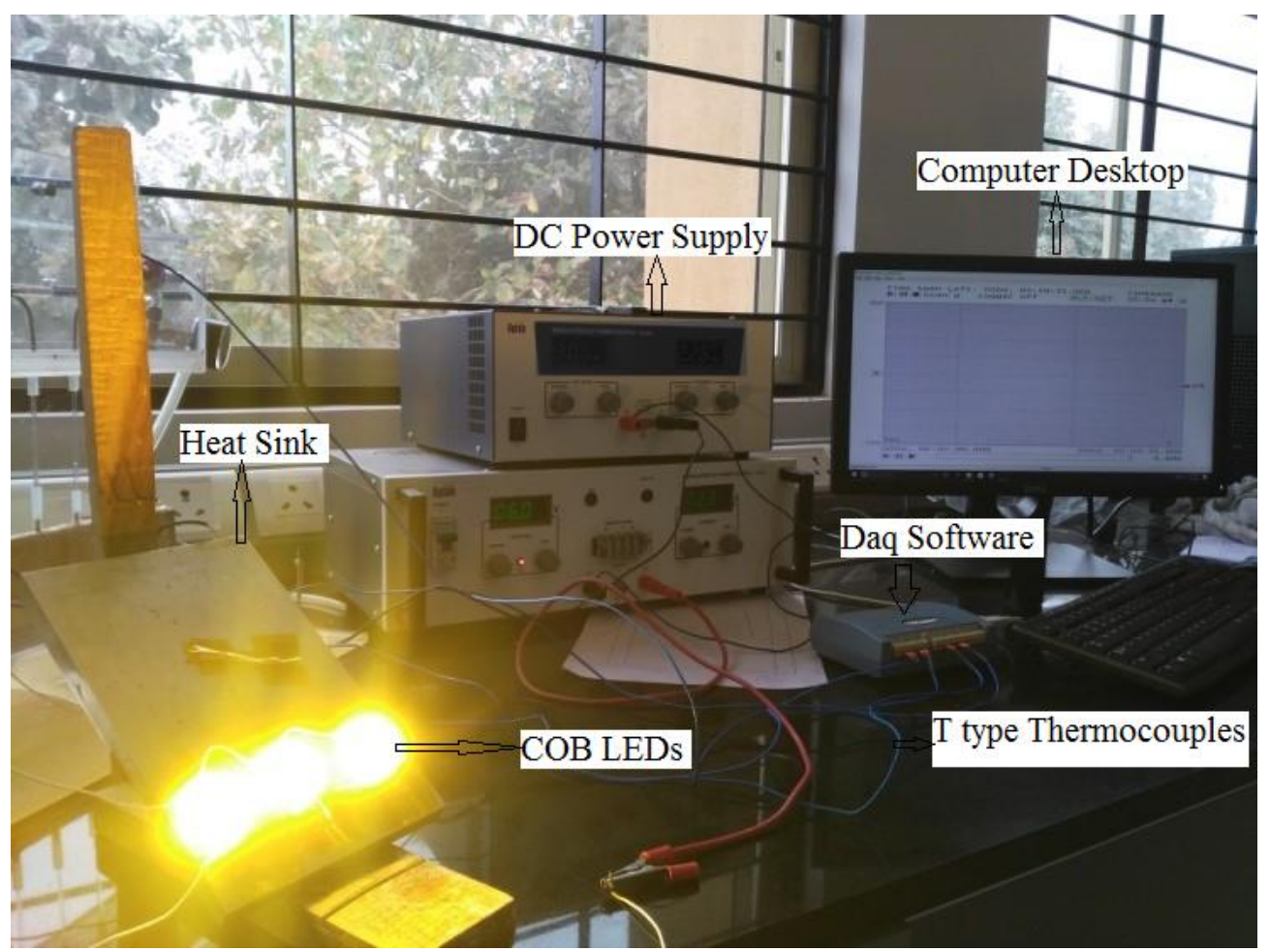

Figure 2. Experimental Setup of the present study

\section{Preparation and Characterization of Nanofluids}

MWCNTs were acquired from Reinste Nano Endeavors Private Ltd. Also concentrated $\mathrm{H}_{2} \mathrm{SO}_{4}$, concentrated $\mathrm{HNO}_{3}$ and $\mathrm{Cu}$, and $\mathrm{Na}_{2} \mathrm{SO}_{3}$ were gotten from Merck. The crude MWCNTs and copper oxide were sonicated for 4 hours at $80^{\circ} \mathrm{C}$ of an ultrasonic shower with a $(\mathrm{v} / \mathrm{v}, 3: 1)$ blend of concentrated $\mathrm{H}_{2} \mathrm{SO}_{4}$ and $\mathrm{HNO}_{3}$ to present oxygen containing useful gatherings on the crude MWCNT surface and it was sifted and washed over till the washing demonstrated no acridity.

The clean MWCNTs were dried in the stove at $80^{\circ} \mathrm{C}$ for 24 hours and the process was repeated for copper oxide. The MWCNT and CuO/MWCNT catalysts were described utilizing a wide range of systematic analytical techniques, including $\mathrm{X}$ - ray diffraction (XRD), scanning electron microscopy with energy dispersive X-ray spectroscopy (SEM-EDS).

These investigations were led to build up a better understanding of the physico - chemical properties of these catalysts. In order to investigate the morphology and the chemical composition of the materials, SEM-EDS analysis was also carried out to evaluate the elemental composition of nanofluids. The morphology of MWCNT and CuO-MWCNT samples was analyzed using SU $3500 \mathrm{~N}$ emission scanning electron microscopy (SEM) operating at $10 \mathrm{kV}$. Figures $3 \mathrm{a}$ and $3 \mathrm{~b}$ show the SEM images of MWCNT and CuO-MWCNT respectively. From the SEM image of MWCNTs (Figure 3a), a tubular network is observed. 


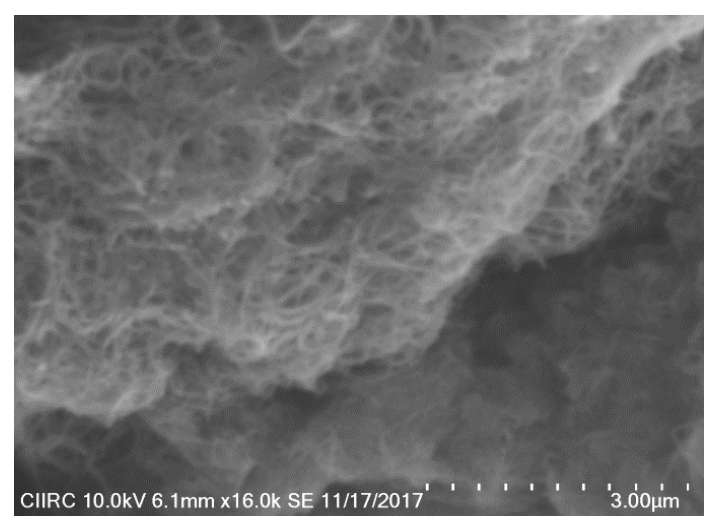

(a)

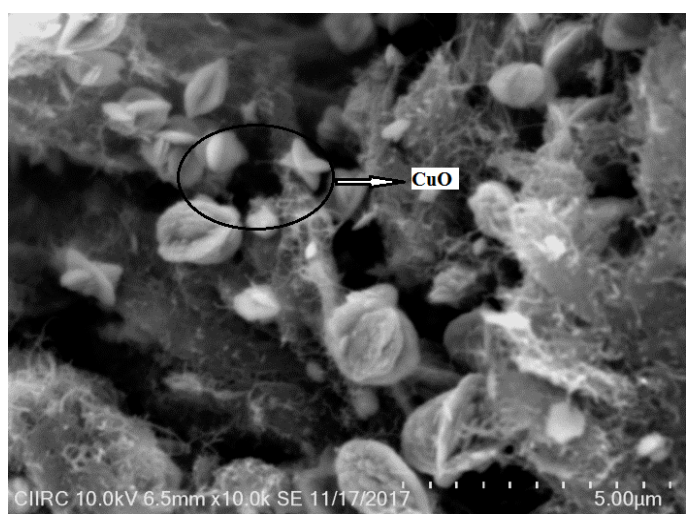

(b)

Figure 3. SEM image of (a) MWCNT and (b) CuO-MWCNT

In case of $\mathrm{CuO}-\mathrm{MWCNTs}$, the formed $\mathrm{CuO}$ flakes appear to have agglomerated to form of flowerlike morphology within the MWCNTs network. EDS spectra indicate the presence of $\mathrm{Cu}, \mathrm{C}$ and $\mathrm{O}$ in the case of CuO-MWCNTs and only $\mathrm{C}$ and $\mathrm{O}$ in the case of MWCNTs as shown in Figure 4a and $4 b$.

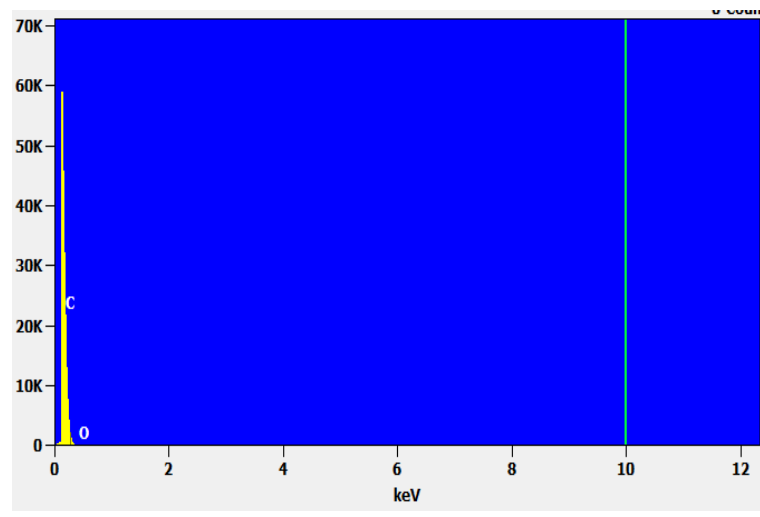

(a)

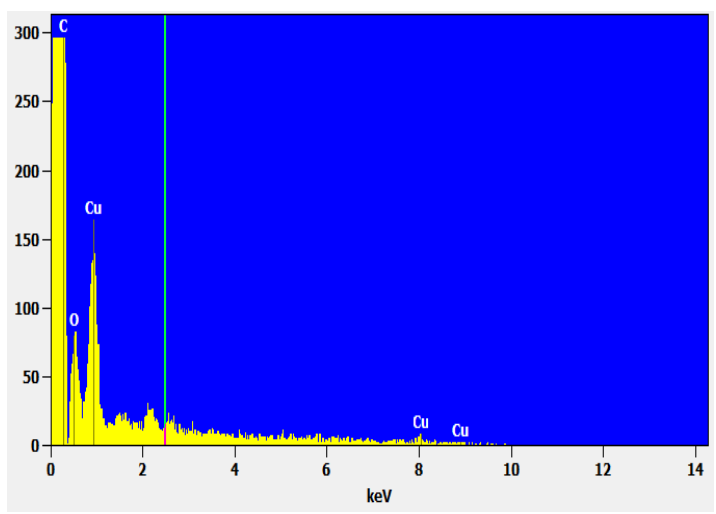

(b)

Figure 4. EDS spectra of (a) MWCNT and (b) CuO-MWCNT

Figures 5a and 5b show the P-XRD patterns of MWCNT and CuO-MWCNT respectively. Bruker D2 Phaser XRD system was used to get X-ray diffraction (XRD) patterns. The P-XRD patterns of MWCNT exhibit two sharp peaks at $2 \Theta$ values $26^{\circ}$ and $42^{\circ}$ corresponding to (002) and (100) hkl planes respectively [30]. While the P-XRD patterns of CuO-MWCNT exhibit peaks at $2 \theta=32.51^{\circ}$, $35.53^{\circ}, 38.5^{\circ}, 46.28^{\circ}, 48.76^{\circ}, 53.58^{\circ}, 58.31^{\circ}, 61.58^{\circ}, 66.24^{\circ}$ and $68.08^{\circ}$ corresponding to different hkl planes of monoclinic phase of $\mathrm{CuO}[31,32]$. It is noticed that the intensity of the peak at $26^{\circ}$ corresponding to (002) plane of MWCNT is less in CuO-MWCNT compared to other peaks. These might be due to the low MWCNT content. 


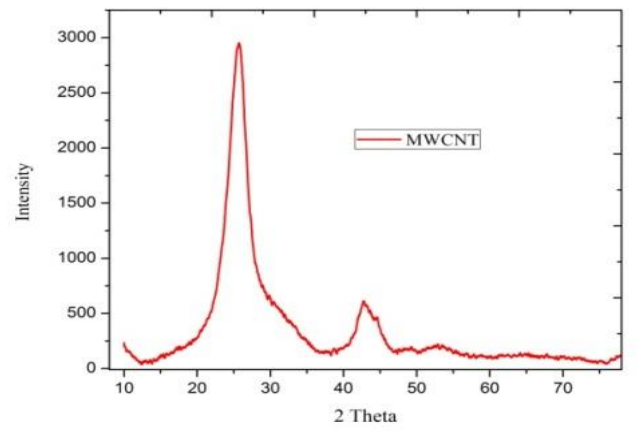

(a)

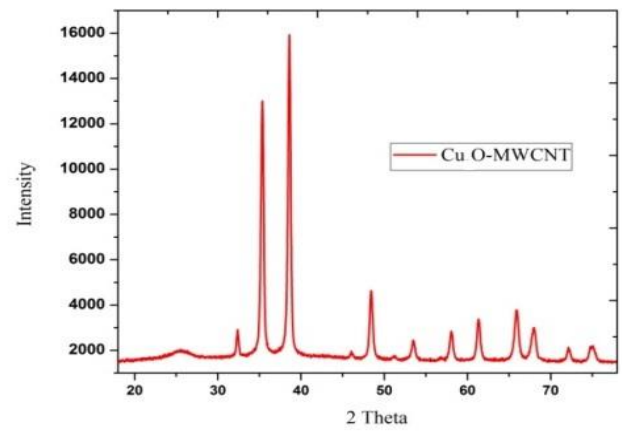

(b)

Figure 5. XRD spectra of (a) MWCNT and (b) CuO-MWCNT

\section{RESULTS AND DISCUSSION}

\section{Effect of Liquid Cooling for Enhanced Heat Transfer}

The experiment was done using de-ionized water as the cooling fluid for $100 \%$ fill ratio to investigate the thermal enhancement of LED with liquid as the cooling medium. Results were compared with the conventional heat sink to determine the influence of liquid cooling for enhanced heat transfer. The junction temperature of the LED with heat sink, in the presence of liquid exhibits, was in agreement as presented in Figure 6. It evidently depicts that minimized junction temperature is obtained with the use of liquid cooling in the heat sink compared to the conventional heat sink. Fluid pockets in the heat sink efficiently strengthen the convective heat transfer. It demonstrates that conventional cooling technique reduces the junction temperature but requires time to dissipate it to the atmosphere. The junction temperature of the LED increased rapidly without cooling fluids and attained $210^{\circ} \mathrm{C}[33,34]$.

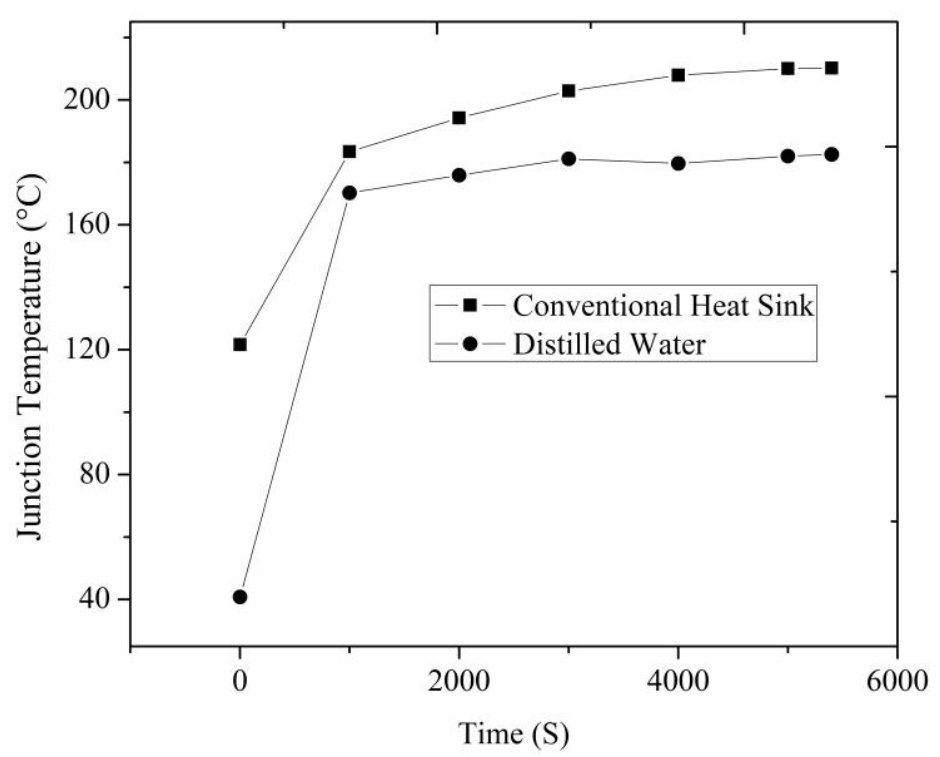

Figure 6. Variation of operating temperature for De-ionized water and conventional heat sink 


\section{Influence of Nanoparticle Volume Fraction on Heat Transfer}

In this section, thermal characterization of nanofluid in terms of reduction in junction temperature of LED is examined experimentally. The effect of volume fraction on heat transfer characterization of MWCNT/De-ionized water and CuO-MWCNT/De-ionized water nanofluids was studied in terms of reduced LED junction temperature. Various suspensions of MWCNT and CuO-MWCNT were tested at a volume fraction of $0.2,0.4,0.6$ and $0.8 \mathrm{wt} \%$. The variation of the junction temperature of LED versus time for different volume fractions of MWCNT and CuO are depicted in Figure 7 and 8. It is evident that the heat dissipation enhances with an increase in volume fraction due to its high thermal conductivity. The thermal conductivity of nanofluids is higher than that of base fluid and there may exist an optimum concentration that maximizes the heat transfer. These corroborate the finding of various authors that thermal conductivity increases with increase in the concentration of nanoparticles. The enhancement of thermal dissipation at a low volume fraction of nanofluid is relatively small. However, with increasing volume fraction of nanoparticles, the enhancement of thermal dissipation due to its ability to extract heat from the junction temperature is significant [35-38]. Also, it can be seen that the reduced junction temperature is an increasing function of volume fraction. However, there is a negligible difference between 0.6 and $0.8 \mathrm{wt} \%$ of total volume which may be because of increase in particles tending to clog up fluid pocket channels.

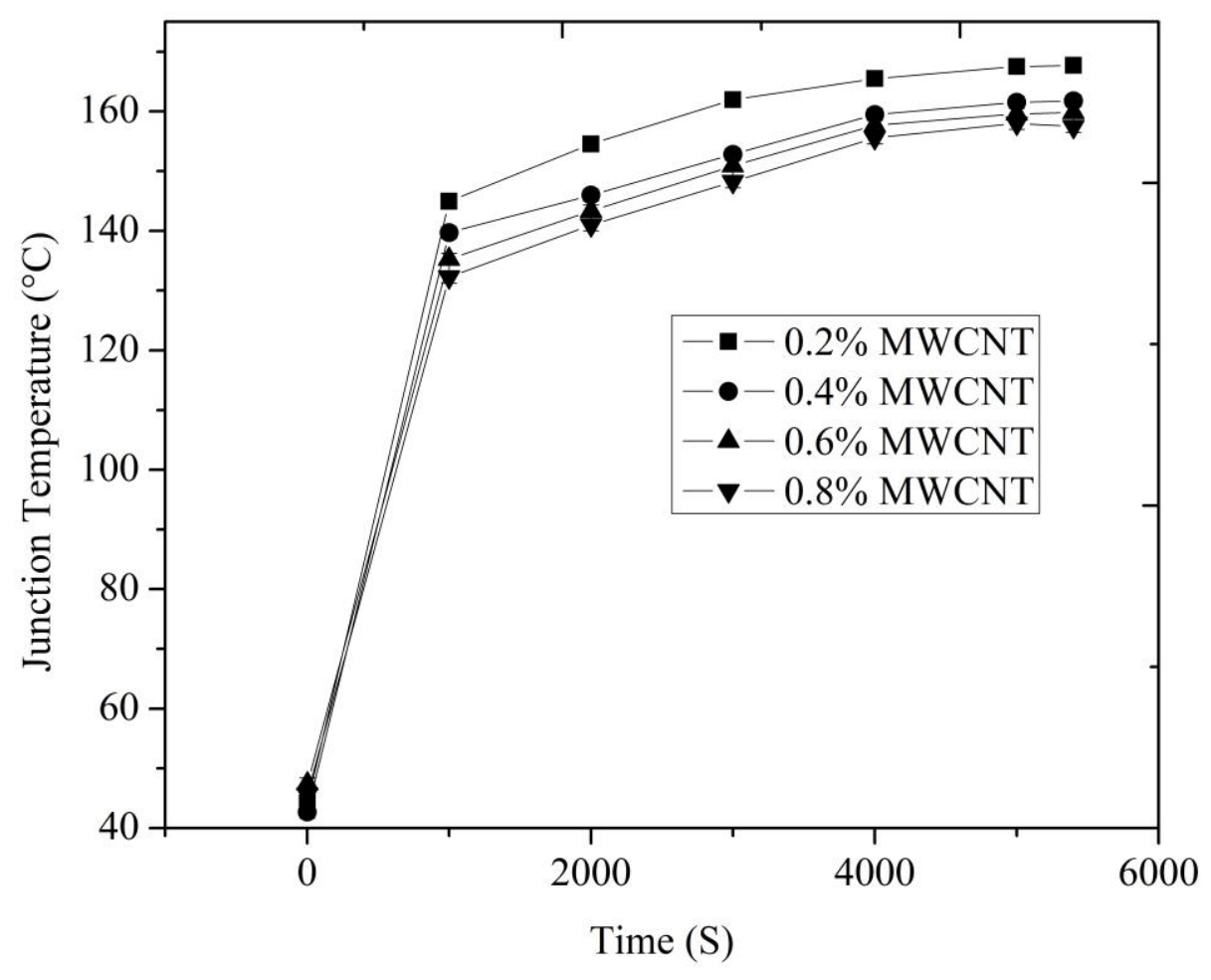

Figure 7. Junction temperature of LED of MWCNT for different volume fraction 


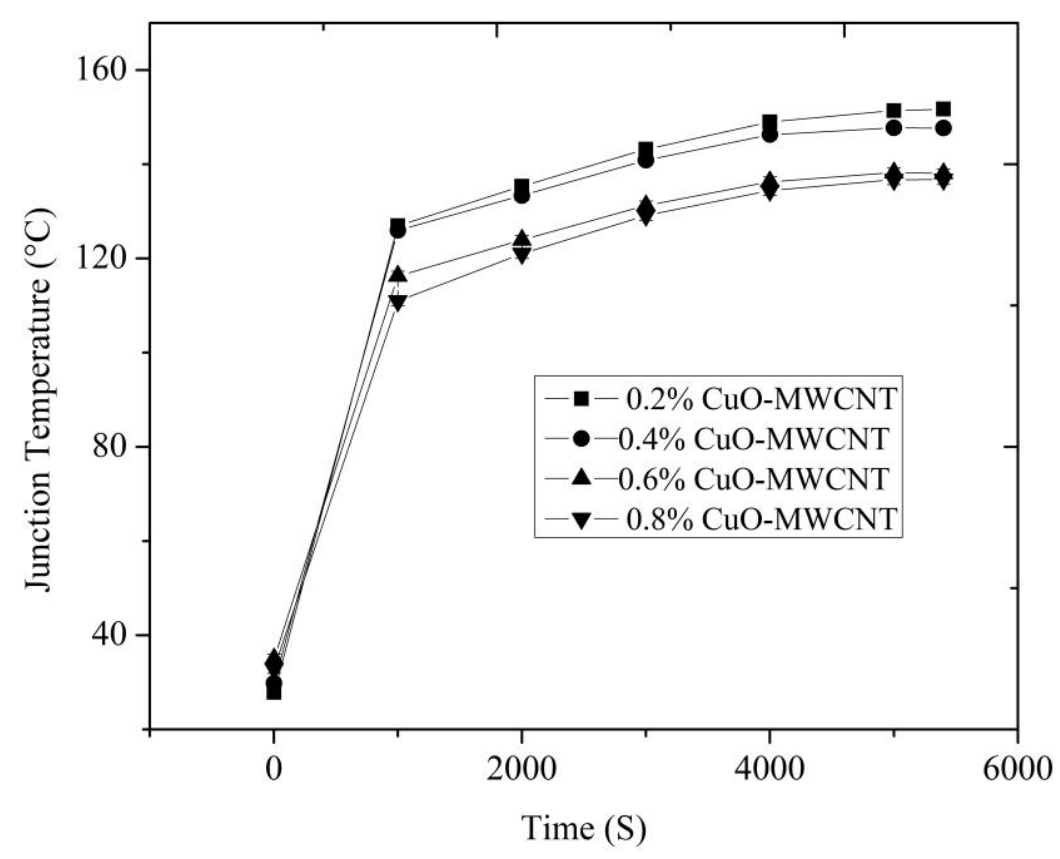

Figure 8. Junction temperature of LED of CuO-MWCNT for different volume fraction

\section{Effect of Nanofluids on Natural Convection}

Natural convection is a passive cooling technique and therefore can be used in many heat transfer applications to improve heat transfer rate without any additional power. Nanofluids evolved over the years due to the limitation of the conventional heat transfer fluids to facilitate higher cooling rates. The use of nanofluids through natural convection mode further enhances the performance and compactness of heat transfer devices. In this study, the HSFP was designed and operated in such a way that the circulation of nanofluids may happen naturally due to rise in junction temperature of LED and return on its own due to the gravity effect. This mechanism tends to achieve a better circulation of fluid within the circular tubes of HSFP. This continued circulation of fluid in the HSFP improved the heat dissipation quickly as compared to the conventional heat sink. The added MWCNT nanofluid with water further enhanced the dissipation due to increase in thermal conductivity; the use of higher thermal conductivity material reduced the thermal resistance significantly. Overall results show that the existing model HSFP for heat dissipation of LED COB is valid for both single and composite nanofluids. It is evident from Figure 9 that there is an enhanced heat transfer rate when composite nanofluids are used due to added nanoparticles of copper. Copper in the MWCNT increases the thermal conductivity of the nanofluid which results in improved heat transfer. The results depict that both single and composite nanofluids are in good agreement. The composite nanofluid exhibited pronounced heat transfer followed by MWCNT nanofluid and liquid cooling using distilled water. The junction temperature increased rapidly and prolonged over a period when the conventional heat sink is used. Passive cooling with distilled water was found to be comparatively good. Liquid cooling is an effective tool where heat dissipation is necessary. Thus, the HSFP proves to be an alternative low-cost device that can be effectively used for cooling of LEDs. 


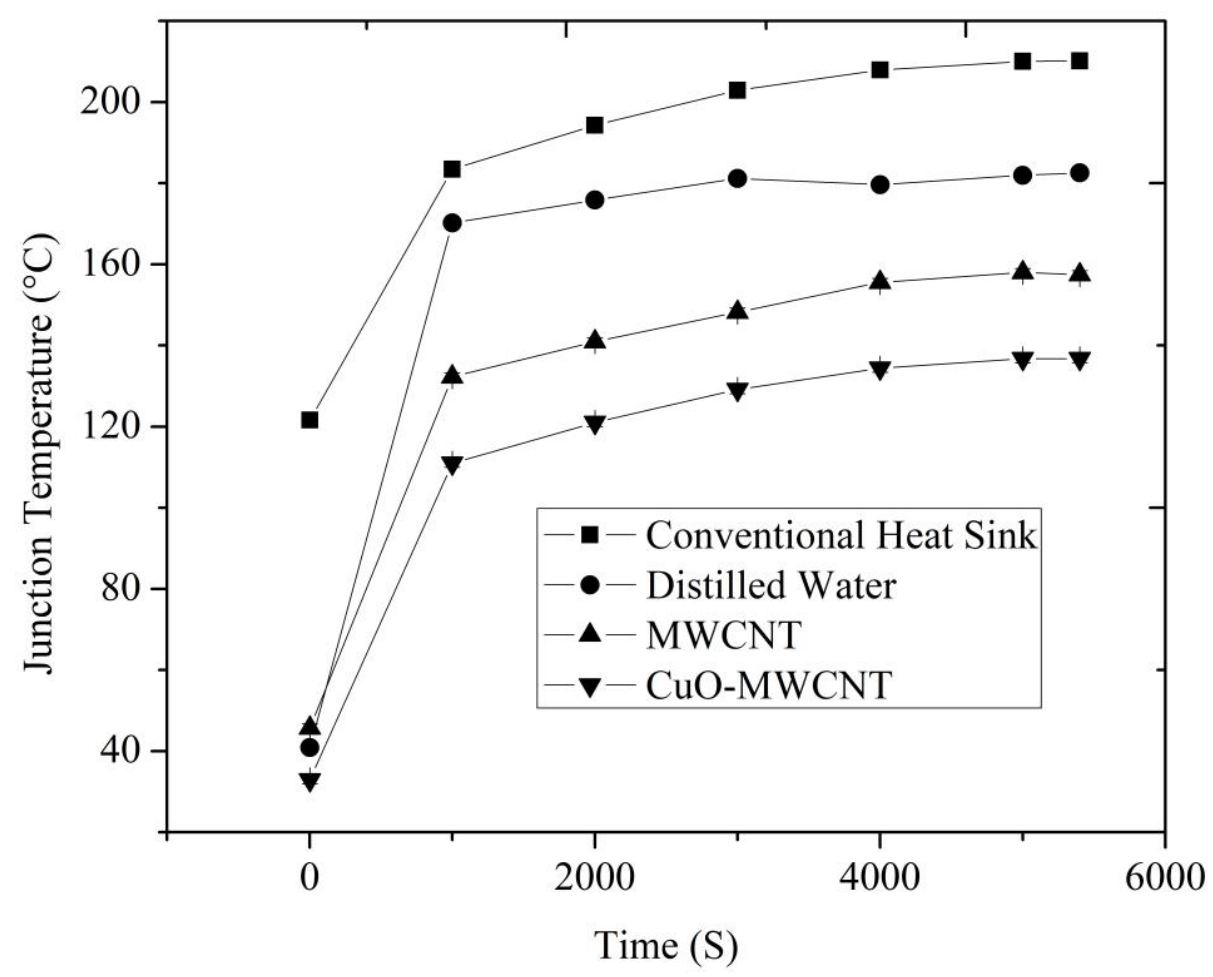

Figure 9. Effect of nanofluids on junction temperature of LED

\section{Thermal Resistance of the Nanofluids}

Thermal resistance analysis was performed to evaluate the efficacy of these nanofluids used as heat transfer fluids. The heat transfer characteristics of the heat sink for various nanofluids were evaluated by the thermal resistance. The overall thermal resistance of LED COB as the ratio of the difference in junction temperature and ambient to the maximum heat load applied to the LED COB was considered as shown in Eq. 1.

It is evident from Figure 10 that thermal resistance increases with increase in junction temperature; it is worthy to note that it reduces with increase in volume fraction of nanoparticles. From the literature, it is observed that the increased nanoparticles volume fraction increases the thermal conductivity of the coolant. Therefore, increase in the thermal conductivity increases the convective heat transfer coefficient which, in turn, reduces the convective thermal resistance and, therefore, the overall thermal resistance of the CuO-MWCNT was found to be higher than the MWCNT nanofluid.

$$
R=\frac{T j-T Q}{Q}
$$




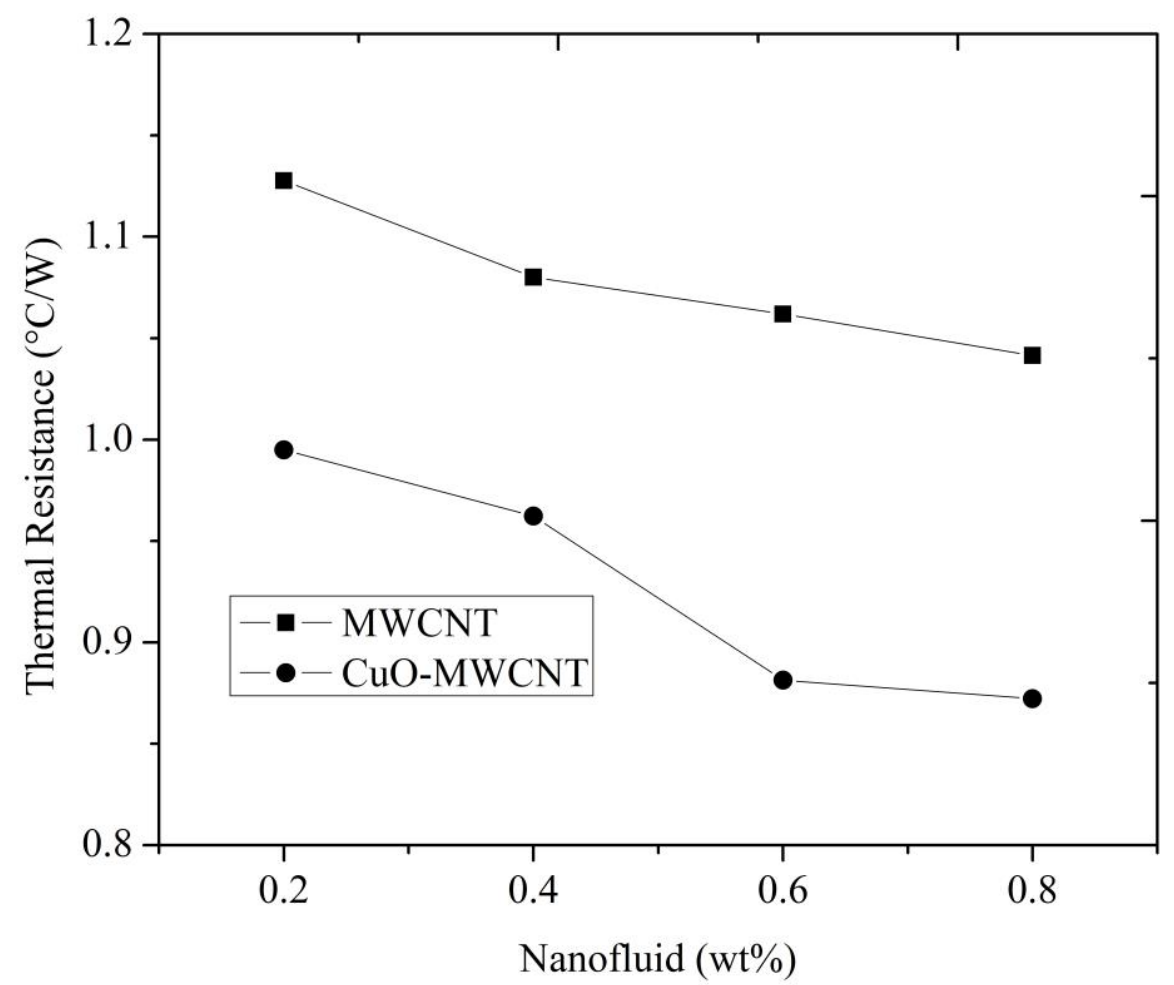

Figure 10. Thermal resistance for varying fill ratio for MWCNT and CuO-MWCNT nanofluids

\section{CONCLUSIONS}

In this work, natural convective heat transfer characterization for MWCNT based nanofluids flowing in fluid pockets within the base of the heat sink was studied. Heat transfer performance studies on natural convection using MWCNT based nanofluids for different concentrations in the range of 0.2 to $0.8 \mathrm{wt} \%$ of the total volume were performed and validated using liquid cooling and a conventional heat sink. The heat transfer effectiveness of the nanofluids was measured in terms of the reduced junction temperature of the LEDs and thermal resistance of the nanofluids. Experimental results demonstrated that the thermal resistance of the LED COB cooling system is reduced by $15 \%$ when using the novel HSFP model with de-ionized water as the heat transfer fluid. The effect of varying nanofluid concentrations with the base fluid enhanced heat transfer in the range of 29\%. The combined effect of MWCNT and CuO-MWCNT further reduced the thermal resistance by $41 \%$. Finding also discloses that between 0.6 and $0.8 \mathrm{wt} \%$, there was no significant change in the result. However, a decline in heat transfer performance was seen especially for conventional heat sinks.

A maximum heat transfer enhancement of $35 \%$ occurred for $0.6-0.8 \mathrm{wt} \%$ volume concentration and there was a negligible difference for further increase in concentration which may be due to clogging up and restricted motion of the fluid. The results of this study are believed to be attributed by the use of MWCNT based nanofluids with buoyancy-driven heat transfer mechanism as a useful technique for enhancing the performance of high power LED particularly street light applications without the aid of external power. From the converged experimental results, it is worthy to note that the feature of HSFP providing longitudinal fluid pockets filled with MWCNT based nanofluids as the cooling agent is significantly useful for the high-power LED package as against the conventional heat sink. Thus, it can be concluded that the use of nanofluids in natural convection mode demonstrated a significant enhancement in their heat transfer performance when used for high 
power LED since they maintained very low junction temperature. However, the volume fraction range of nanoparticles would require more in-depth experimental studies. Therefore, experimental studies are very crucial for understanding the thermophysical behavior of nanofluids.

\section{ACKNOWLEDGEMENTS}

The authors would like to thank the Centre for Incubation, Innovation, Research and Consultancy, Jyothy Institute of Technology for the laboratory and financial assistance provided.

\section{REFERENCES}

[1] Mehmet A, Charles B, Stanton W, and James P. Thermal management of LEDs: Package to System. Conference on Solid State Lighting, Proc. of SPIE. 2004; 5187: 0277-786X/04/15.

[2] Tisha D, Indranil G. Review of micro and mini channel heat sinks and heat exchangers for single phase fluids. Renewable and Sustainable Energy Reviews. 2015;4:1298-1311.

[3] Yiwei W, Jiwen C, Fangming J, Wenjiong C. Heat dissipation of high-power light emitting diode chip on board by a novel flat plate heat pipe. Applied Thermal Engineering. 2017;123:19-28.

[4] Zirong L, Shuangfeng W, Jiepeng H, Yanxin H, Jinjian C, Winston Z, Eton L. Heat transfer characteristics and LED heat sink application of aluminum plate oscillating heat pipes. Applied Thermal Engineering. 2011;31:2221-2229.

[5] Patrice E, Salma H, Thierry M. Thermo physical properties and heat transfer performance of carbon nanotubes water-based nanofluids. Journal of Thermal Analysis and Calorimetry, Springer-Verlag; Springer (Kluwer Academic Publishers). 2017;3:2075-2081.

[6] Hamed KA, Ahmad A, Kazi SN, Chew BT, Badarudin A. Experimental investigation of thermophysical properties and heat transfer rate of covalently functionalized MWCNT in an annular heat exchanger. International Communications in Heat and Mass Transfer. 2016;75:67-77.

[7] Hessam T, Jorge LA, Ehsan ML. Enhanced thermophysical properties of multiwalled carbon nanotubes based nanofluids. Part 2: Experimental verification. 2017;10: 117.

[8] Mohammad HE, Seyfolah S, Wei MY, Masoud A, Nima S. Study on thermal conductivity of water-based nanofluids with hybrid suspensions of $\mathrm{CNTs} / \mathrm{Al}_{2} \mathrm{O}_{3}$ nanoparticles. J Therm Anal Calorim. 2016;124:455-460.

[9] Wail SS, Ahmad A, Kazi SN, Badarudin A. Stability and thermo physical properties of noncovalently functionalized graphene nano platelets nanofluids. Energy Conversion and Management. 2016;116:101-111.

[10] Munkhbayar B, Md RT, Jinseong J, Hanshik C, Hyomin J. Surfactant-free dispersion of silver nanoparticles into MWCNT-aqueous nanofluids prepared by one-step technique and their thermal characteristics. Ceramics International. 2013;39:6415-6425.

[11] Aravind SSJ, Tessy TB, Sabareesh RK, Sumitesh D, Ramaprabhu S. Investigation of structural stability, dispersion, viscosity, and conductive heat transfer properties of functionalized carbon nanotube based nanofluids. J. Phys. Chem. 2011;115:6737-16744.

[12] Vanaki SM, Ganesan P, Mohammed HA. Numerical study of convective heat transfer of fluids: A Review. Renewable and Sustainable Energy Reviews. 2016;54:1212-1239.

[13] Mohammadali B, Alimorad R, Davood R, Roghayeh L, Azadeh A. Synthesis of spherical silica/multiwall carbon nanotubes hybrid nanostructures and investigation of thermal conductivity of related nanofluids. Thermochimica Acta. 2012;549:87-94. 
[14] Kouloulias K, Sergis A, Hardalupas Y. Sedimentation in nanofluids during a natural convection experiment. International Journal of Heat and Mass Transfer. 2016;101:11931203.

[15] Zeinali H, Esfahany MN, Etemad SG. Experimental investigation of convective heat transfer of $\mathrm{Al}_{2} \mathrm{O}_{3}$ /water nanofluid in circular tube. International Journal of Heat and Fluid Flow. 2007;28; 203-210.

[16] Gianluca P, Samuel P, Mihir S. Nanofluids and their properties. Applied Mechanics Reviews. 2011;64:031002-1.

[17] Murshed SMS, Leong KC, Yang C. Thermophysical and electrokinetic properties of nanofluids - A critical review. Applied Thermal Engineering. 2008;28:2109-2125.

[18] Yulong D, Hajar A, Dongsheng W, Richard AW. Heat transfer of aqueous suspensions of carbon nanotubes (CNT nanofluids). International Journal of Heat and Mass Transfer. 2006;49:240-250.

[19] Smail H, Ahmed J, Revo S, Ivanenko K, Youcef J, Avramenko T. Thermal analysis of copper-titanium multiwall carbon nanotube composites. Nanoscale Research Letters. 2017;12:251.

[20] Majid Z, Arash K, Davood T. Experimental study of the effect of solid volume fraction and Reynolds number on heat transfer coefficient and pressure drop of $\mathrm{CuO}-$ Water nanofluid. Experimental Thermal and Fluid Science. 2016;76:342-351.

[21] Saeedinia M, Akhavan-Behabadi MA, Razi P. Thermal and rheological characteristics of $\mathrm{CuO}-\mathrm{Base}$ oil nanofluid flow inside a circular tube. International Communications in Heat and Mass Transfer. 2012;39:152-159.

[22] Ali H, Naveed R, Asim U, Ayyaz A, Hina M. Enhancement of convective heat transfer coefficient of ethyleneglycol base cuprous oxide $\left(\mathrm{Cu}_{2} \mathrm{O}\right)$ nanofluids. Heat and Mass Transfer. 2018;54:325-332.

[23] Hooman Y, Nurin WMZ, Samira G, Seyed F, Seyed S, Abdullah AAAA, Mohamad AA, Mahidzal D, Kazie SN. Convective heat transfer enhancement with graphene nanoplatelet/ platinum hybrid nanofluid. International Communications in Heat and Mass Transfer. 2017;88:120-125.

[24] Hong SJ, Seongpil A, Hyun GP, Min-Woo K, Salem S. Al-Deyab, James SC, Jeehoon C, Sam SY. Enhancement of critical heat flux and superheat through controlled wettability of cuprous-oxide fractal-like nanotextured surfaces in pool boiling. International Journal of Heat and Mass Transfer. 2017;107:105-111.

[25] Ellahi R, Hassan M, Zeesha A. Study of natural convection mhd nanofluid by means of single and multiwalled carbon nanotubes suspended in a salt water solution. IEEE Transactionson Nanotechnology. 2015;14:726-734.

[26] Heris SZ, Esfahany MN, Etemad SG. Experimental investigation of convective heat transfer of $\mathrm{Al}_{2} \mathrm{O}_{3} /$ water nanofluid in circular tube. International Journal of Heat and Fluid Flow. 2007;28:203-210.

[27] Garbadeen ID, Sharifpur M, Slabber JM, Meyer JP. Experimental study on natural convection of MWCNT-water nanofluids in a square enclosure. International Communications in Heat and Mass Transfer. 2017;88:1-8.

[28] Muryam H, Ramzan N, Umer A, Awan GH, Ali Hassan. Comparative study of convective heat transfer characteristics of nanofluids. Heat Mass Transfer. 2017;53:2309-2316.

[29] Bailin F, Pei Z, Ganghan H, Jun WY. Research on properties of fluid pressure drop for electric vehicle igpt pin fin heat sink. International Journal of Engineering, Transaction A:Basics. 2015; 28:627-633.

[30] Monaheng LM, Lebea NN, Soraya PM, Edward NN, Sabelo DM. Chitosan-based nanocomposites for de-nitrification of water. Physics and Chemistry of the Earth. 2017;100:113. 
[31] Manyasree D, Kiran MP, Ravikumar R. CuO nanoparticles: Synthesis, Characterization and their bactericidal efficacy. Int J App Pharm. 2017;9:71-74.

[32] Prachi K, Surapaneni M, Swati C, Padmavathy N. Understanding the pathway of antibacterial activity of copper oxides nanoparticles, electronic supplementary material (esi) for rsc advances. The Royal Society of Chemistry. 2015;5:12293-12299.

[33] Sangmesh, Gopalakrishna KN, Manjunath SH, Krishna V, Keertishekar MS. Thermal performance of heat sink with fluid pockets for high power light emitting diode, International Journal of Automotive and Mechanical Engineering. 2017;14(4): 4846-4862.

[34] Lv L, Li J, Zhou G. A robust pulsating heat pipe cooler for integrated high power LED chips. Heat and Mass Transfer. 2017;53:3305-13.

[35] Abidin SZ, Mohamad IS, Hashim BAY, Abdullah N, Hafiz MIM, Masripan NAB, Abdullah A. Investigation of thermal characteristics of CNF-based nanofluids for electronic cooling applications. Journal of Mechanical Engineering and Sciences. 2016;10(3):2336-2349.

[36] Kou HS, Lee JJ, Chen CW. Optimum thermal analysis of a heat sink with various fin crosssections by adjusting fin length and cross-section. Heat Transfer Engineering. 2008; 29:53745.

[37] Hussein AM, Bakar RA, Kadirgama K, Sharma KV. Experimental measurements of nanofluids thermal properties. International Journal of Automotive and Mechanical Engineering. 2013;7:850-63.

[38] Abdul Hamid K, Azmi WH, Mamat R, Usri NA, Najafi G. Effect of temperature on heat transfer coefficient of titanium dioxide in ethylene glycol-based nanofluid. Journal of Mechanical Engineering and Sciences. 2015;8:1367-75. 